\title{
ASPECTOS SOCIOCULTURALES DE LA PRECARIEDAD LABORAL Y DE SU IMPACTO
}

\author{
PILAR ISLA PERA* \\ *Licenciada Enfermería. Profesora Dpto. de Enfermería. Universidad de Barcelona.
}

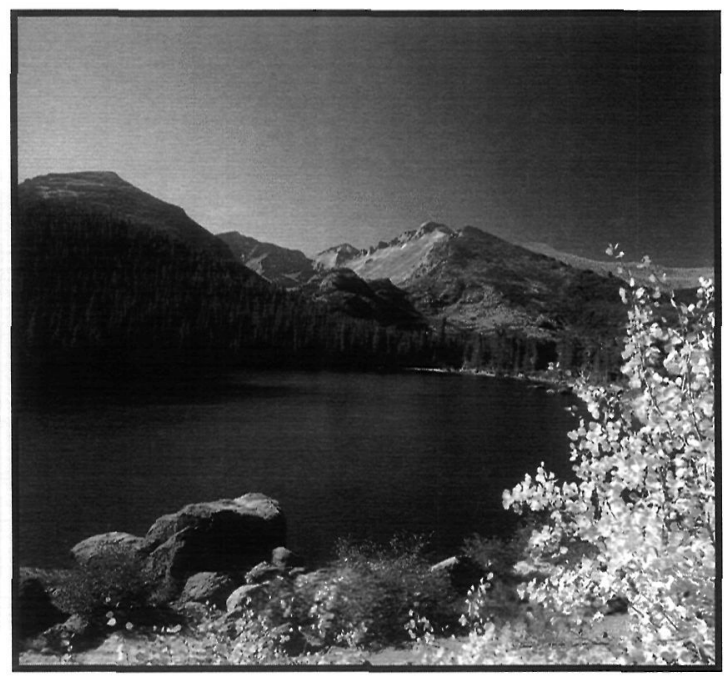

WORK ISSUES ON THE JOB SHORTAGE AND ITS IMPACT

\section{SUMMARY}

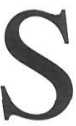
igerist stated, back in 1940, that disease is nothing but life under abnormal conditions. Nowadays millions of people suffer the drama of unemployment and job shortage and this compromises not only their ability to satisfy their primary needs, but also their personal dignity. Nevertheless, the concept of "work" is a profoundly historical category that has evolved to acquire different meanings, to the point of being considered an individual and social goal, the only means to promote oneself and relate to others, so that being "unemployed" is associated with personal and social degradation that can lead to the social discrimination of the unemployed and affect his or her physical, psychological and social health.
This essay analyzes the current work crisis, its individual and social influence and its implications on human development and health. It discusses the main cultural perspectives on the notion of work and presents the hypothesis of a possible influence of the cultural meaning of the concept of "work" on diminishing self-esteem and mental health problems among unemployed people. The essay also highlights possible implications of the current situation in the future taking into consideration socioeconomic and political aspects as well as global demographic growth. Finally, the essay discusses the role that Public Health should have not only in the prevention and control of health problems among the unemployed, but also in the social, economic and cultural changes that are considered necessary to mitigate the serious social differences and to improve the current situation.

\section{INTRODUCCIÓN}

El paro y el subempleo son dramas cotidianos que afectan a millones de personas. La precariedad en el trabajo afecta muy negativamente a la salud de los trabajadores y aumenta el riesgo laboral. Numerosos trabajos han puesto de manifiesto que el desempleo se asocia con peores conductas de salud y mayor morbilidad y mortalidad. También se ha observado que la inestabilidad laboral se asocia con una mayor exposición a riesgos laborales y afecta a la salud mental.

El trabajo se ha convertido en un fin y en un medio y su pérdida pone en peligro la capacidad de satisfacer las necesidades personales y familiares, quebranta la dignidad personal, hace perder la identidad y el sentido de pertenencia y destruye las relaciones sociales.

Pero esto no ha sido siempre así, la noción actual de trabajo se consolidó en el siglo XVIII, 
cuando las nociones de producción y de trabajo se reforzaron mutuamente y se les otorgó un sentido utilitario que permitía identificarlas con un avance inequívoco hacia la felicidad y el progreso.

La economía, en lugar de combatir la escasez, ha favorecido que esta se extendiera y se agravara y que llegara incluso a alcanzar al propio trabajo que es el único instrumento que tiene la gente para su subsistencia y para hacer frente a la sociedad de consumo. Las desigualdades sociales son cada vez mayores de tal forma que la sociedad de hombres libres e iguales que predicaba el liberalismo, se ha convertido en una utopía.

Además de las consecuencias económicas derivadas del paro laboral la pregunta que se plantea es si la concepción simbólica y cultural del trabajo puede disminuir la autoestima del individuo desempleado y afectar a su salud mental. En este trabajo se pretende reflexionar sobre el propio concepto del trabajo, su simbología y significado desde una perspectiva histórica y cultural y recoger las diferentes visiones sobre la crisis actual y las expectativas de futuro.

\section{EL TRABAJO COMO CONCEPTO}

La noción actual del trabajo es una categoría profundamente histórica. En las sociedades primitivas no existía el concepto actual del trabajo, no había distinción entre las actividades productivas y el resto. El tiempo que utilizaban los cazadores recolectores para el aprovisionamiento es muy inferior a la jornada laboral. Marshall Sahlins resalta que la escasez no es intrínseca de los medios técnicos sino que su percepción nace de relacionar medios con fines y que los medios técnicos disponibles en las sociedades primitivas, les permitían cubrir con mucha mas holgura sus fines que lo que ocurre en las sociedades tecnológicas de hoy en día, estando, por lo tanto, más cerca de la abundancia aquellas que estas. Esto se debía al hecho de que no existía el afán de acumular riquezas; para estas sociedades los stocks de riquezas estaban en la naturaleza y no tenía sentido acumularlos.

En la antigüedad clásica, Grecia despreciaba el trabajo manual. Se consideraban actividades libres aquellas que se realizaban por el placer mismo de ejercitarlas como la filosofía, la política, las artes o el deporte. Se consideraba indigno de personas libres desarrollar sus capacidades para obtener una ganancia. Roma continuó despreciando las tareas rutinarias y penosas relacionadas con el aprovisionamiento y la subsistencia. Cicerón escribió: Cuanto tenga que ver con un salario es sórdido e indigno de un hombre libre, porque el salario en esas circunstancias es el precio de un trabajo y no de un arte.

El cristianismo también despreció lo que hoy concebimos como trabajo considerándolo un castigo fruto de una maldición divina, pero con el predominio del capitalismo, le otorgó una creciente veneración. La búsqueda de la salvación por el trabajo y otras prácticas mortificadoras fue recogida por Lutero y los calvinistas que predicaron el ascetismo intramundano. En el siglo XVI, el trabajo se erigía en valor supremo al que debía plegarse la existencia del hombre. Se identificó al trabajo con actividad y al ocio se le asignó un carácter pasivo anulando el significado anterior de esta palabra que se refería también a un ocio activo y creador.

La noción actual de trabajo se consolidó en el siglo XVIII. Las líneas maestras del contexto que hizo progresar la razón productivista se podrían resumir de la siguiente forma: Se extendió entre la población un afán de acumular riquezas levantándose el veto moral que antes pesaba sobre ello. Se modificó la propia noción de riqueza dándole una noción unificada y monetaria que posibilitara la acumulación. Fue preciso que el hombre se creyera capaz de producir riquezas y finalmente, que se postulara que el trabajo era el instrumento básico de esa producción de riquezas.

\section{CRISIS DEL TRABAJO}

En el siglo XIX se produce la hegemonía de un nuevo factor de producción: el capital y esto produce una transformación en la concepción del trabajo. El sistema económico se centra en los valores pecuniarios y se aísla de los valores sociales. Al centrarse en los valores materiales, el salario se convirtió en el único criterio que diferenciaba lo que es trabajo de lo que no lo es, por ejemplo, no se consideró trabajo las actividades realizadas por el ama de casa, mientras si se consideró trabajo el servicio doméstico remunerado. 
Cada vez cobraba mas fuerza la consideración del trabajo como la meta individual y social, hasta tal punto que los pobres pasaron de pedir pan, a pedir trabajo y los burgueses "insolidarios" se convirtieron en bienhechores "creadores de puestos de trabajo".

De esta manera el trabajo se convirtió en el único medio para relacionarse y promocionarse en el ámbito económico, personal y social y perdió fuerza la familia, el grupo, la tribu, o el gremio. Por este motivo, el estar en paro se acompaña de una degradación personal y social que puede desencadenar en la marginación social del parado y afectar su salud física, psicológica y social.

Por otra parte, se asocia el nivel de prestigio de un individuo con su puesto de trabajo y su nivel de riqueza, y se origina una competitividad y una insatisfacción crónica generalizada. Además, la sociedad de consumo ha creado un sin fin de necesidades que cambian y aumentan constantemente. En relación con estos hechos, Ivan Illich afirmaba que "el homo economicus ha servido de eslabón intermedio en la transformación del homo sapiens en homo miserabilis".

En los últimos años la clase obrera se ha visto minada, desbordada, por una transformación sociológica profunda de la estructura de la distribución salarial. Esta desposesión pasó por dos etapas: La primera, marca el paso de la sociedad industrial a la sociedad salarial y la segunda es el producto de la disgregación de esta sociedad cuyas consecuencias se hicieron visibles a mediados de los años 70.

Si se analiza la situación francesa, se observa cómo en 1936, los obreros tenían fuerza, ideología, sindicatos y partidos políticos propios. Los obreros asalariados representaban el $60 \%$ de la población asalariada y el $75 \%$ si se incluyen a los obreros agrarios. En 1975, se mantuvo el número de obreros, pero se produjo una transformación cualitativa en la estructura de la población asalariada. Aparecieron las profesiones intermedias y los cuadros medios y superiores, con ingresos y posiciones más elevados. Ahora la conflictividad social se redistribuye y cada categoría presenta sus propias reivindicaciones. Hasta los años $70 \mathrm{y}$, pese a la pérdida de fuerza de los obreros, se había conseguido una mejora general de las condiciones de vida de todas las categorías obreras y una relativa cohesión entre las categorías, debido a la existencia de unos estatutos bastante estables y homogéneos que, unido a un periodo de pleno empleo en el que el acceso al trabajo parecía asegurado, permitía el poder hablar de un estatuto social mínimo garantizado.

A partir de los años 70 este marco se modifica. Se multiplican las situaciones de trabajo que están más allá de este estatuto social mínimo garantizado y se rompe la solidaridad intracategorial debido a que existen dos grandes riesgos: el paro y la precariedad laboral. Los obreros entran en competencia entre iguales al ver peligrar su puesto de trabajo. Se le pide al trabajador que se movilice a sí mismo para afrontar los nuevos problemas. Ulrich BecK denomina a esta fase "la desestandarización del trabajo", el recurso a estrategias individuales más que a estrategias colectivas para afrontar la situación actual.

Según datos del Instituto Estadístico de la Comunidad de Madrid (1999), las tasas de desempleo en las regiones europeas presentan un amplio abanico. Según datos correspondientes a 1999 el rango variaba de 1,2\% de paro en la región finlandesa de Aländ, al 28,7\% en la italiana Calabria. Tras esta se colocaban algunas regiones españolas: Andalucía con el $26,8 \%$ y Extremadura, Ceuta y Melilla con un $25,5 \%$.

En España, durante el año 2000, sin contar el mes de diciembre, el $92 \%$ de los contratos registrados en el INEM fueron temporales y más de la mitad de los contratos indefinidos contenían elementos de precariedad: el 19,3\% eran indefinidos a tiempo parcial y el 37,2\% correspondía al modelo de fomento de la contratación indefinida que contempla una menor indemnización por despido improcedente que el despido ordinario, circunstancia que ha contribuido a que se rescindieran el $30 \%$ de estos contratos desde su entrada en vigor en 1997.

El desempleo y la precariedad laboral tienen importantes repercusiones sociales y en la actualidad constituyen un problema económico y político de gran envergadura. Francisco Checa Olmos en su trabajo "Reflexiones antropológicas para entender la pobreza y las desigualdades sociales" une conceptos de miseria, injusticia y violencia con el desempleo o los sin trabajo "involuntarios". La crisis económica que priva de empleo a millones de 
personas rompe con los valores sociales o morales. Los jóvenes ven alargada su etapa infantil, constituyen lo que se ha llamado "la generación Peter Pan", deben vivir durante mucho más tiempo con los padres y sienten como se les roba espacio y protagonismo. Este problema no afecta a todos por igual y son las personas con menos recursos, no sólo económicos, sino también culturales y sociales, los que más sufren las consecuencias.

\section{DESEMPLEO Y PRECARIEDAD LABO- RAL. NATURALEZA Y MEDICIÓN}

Cuando se quiere describir las actuales condiciones del mercado laboral, se utiliza frecuentemente el término precariedad pero resulta impreciso ya que se utiliza tanto para describir un trabajo temporal, como una situación de paro, o unas condiciones especiales de trabajo. Esta falta de definición es un obstáculo para diseñar estudios rigurosos que sean comparables. Amable y Benach plantean cuatro dimensiones relevantes del concepto precariedad: temporalidad; vulnerabilidad; nivel salarial y posibilidad de acceso a ciertos beneficios sociales de la seguridad social y del seguro de desempleo.

Para describir la situación del empleo en una sociedad, se utiliza la "tasa de desempleo": proporción de trabajadores en paro en relación a la población activa, pero esta cifra es demasiado inexacta debido al sesgo de la definición de "persona en paro" y "persona activa".

Se llama población activa al conjunto de personas de una sociedad, que estando en edad de trabajar y estando capacitados para hacerlo, tienen o desean tener un empleo remunerado. Los miembros de este grupo que están buscando trabajo y no lo encuentran constituirán los trabajadores en paro. Quedan excluidos de la población activa los menores de edad, los jubilados, las personas con incapacidad, los estudiantes, las amas de casa y los que no buscan trabajo.

En España hay dos formas de recoger los datos de desempleo. Las oficinas de empleo consideran en paro a los trabajadores incluidos en sus ficheros que esperan un trabajo. Las estadísticas que elaboran se llaman "paro registrado". El Instituto Nacional de Estadística realiza mensualmente y publica una Encuesta de Población Activa (EPA) cuyos resultados reciben el nombre de "paro declarado". Ambos métodos pueden contener importantes errores: Pueden infravalorar el problema del paro ya que no incluyen el subempleo (trabajadores a tiempo parcial o periodos cortos) y los que no se presentan en las oficinas de empleo.

\section{DESARROLLO HUMANO. SITUACIÓN MUNDIAL}

Con Smith, Ricardo y Marx, el trabajo se erigió en el principal factor de producción de riqueza. Smith escribió, en 1776: El trabajo anual de cada nación es el fondo que la surte originariamente de todas las cosas necesarias y útiles para la vida que se consumen anualmente en ella.

Desde entonces hasta ahora las teorías económicas sobre el trabajo no han sido capaces de proporcionar riqueza y evitar la miseria en gran parte del planeta, sino que continuamente crecen las desigualdades sociales. En el mundo existen grandes diferencias que condicionan la posibilidad de vivir. Hay una distribución desequilibrada de los recursos que permite que el $20 \%$ de la población disfrute del $80 \%$ de los recursos que se producen y se consumen. Este hecho, es por si sólo indignante puesto que conlleva la miseria, el hambre y la muerte de una gran parte de la población.

Si se toman los informes de la ONU sobre el índice de desarrollo humano en la década de los 90 se puede apreciar con datos objetivos las desigualdades existentes en el planeta como, por ejemplo, el hecho de que la quinta parte más rica detenta casi el $85 \%$ del PIB mundial, mientras que la quinta parte más pobre apenas si pasa del 1\%. 1.300 millones de personas están viviendo en la pobreza más absoluta con menos de 1 dólar diario. 1.000 millones no disponen de agua potable. 4.000 millones de personas no ven cubiertas diariamente sus necesidades básicas.

La pobreza humana es sólo una cara de la penuria humana, la otra cara la conforman la persistencia de las desigualdades sociales. Las desigualdades son igualmente grandes dentro de los propios países: En Brasil el 50\% de la población más pobre recibía, en 1960, el 18\% de los ingresos nacionales y en 1995 sólo el 11,6\%. El 10\% más rico recibía, 
en 1960 , el 54\% de los ingresos nacionales, y en 1995 el 63\%. En los países industrializados también existen grandes diferencias: en Rusia la proporción de ingresos en el 20\% más rico es 11 veces más alta que en el 20\% más pobre. En Australia y en Gran Bretaña la proporción es de 10 veces.

El caos económico amenaza la salud de todos los países al no poder aplicar medidas preventivas ni curativas. El VIH/SIDA ha segado más de 12 millones de vidas desde su inicio hace 18 años y algunos expertos afirman que sólo ha alcanzado el $10 \%$ de su magnitud y que será en el futuro próximo cuando mostrará las consecuencias reales sobre las personas, las sociedades y la economía. El SIDA no sólo afecta a la esperanza de vida al nacer, sino que aparte del drama humano que representa la muerte de las personas, los efectos económicos y sociales en estos países pueden ser catastróficos, dado que la mayor parte de víctimas se producen en la edad más productiva y ello afecta a la sostenibilidad de las familias y de la sociedad. De la misma manera que la pobreza facilita la epidemia, la epidemia intensifica la pobreza.

\section{LA CULTURA DEL TRABAJO. DIFEREN- TES PERSPECTIVAS}

El trabajo también posee una cultura específica que consiste en la forma en que una determinada sociedad concibe y asume el hecho del trabajo humano y posee, también, unas subculturas específicas determinadas por las actitudes, conductas y valoraciones que cada clase o sector social tiene en relación con el trabajo y con los trabajadores.

Victor M. Durán describe la cultura del trabajo en la sociedad latinoamericana desde diferentes puntos de vista. Una de ellas consiste en considerar esta cultura como un modo de ser, como una actitud y conducta personal y colectiva frente al trabajo. Desde este punto de vista admite que existe, en determinados sectores, el estereotipo de que a los latinoamericanos no les gusta trabajar y que esta es la causa de que sus países sean pobres. Si se relaciona esta supuesta cultura del trabajo con el desempleo, significaría que este no es debido a la falta de trabajo, sino a las pocas ganas de trabajar.

Otra forma de contemplar la cultura del trabajo es hacerlo como un sistema de valores. Desde esta perspectiva existen dos culturas muy diferenciadas: la cultura economicista-materialista y la cultura humanista-espiritualista. La primera consiste en valorar el trabajo exclusivamente en términos económicos y materiales. El capitalismo neoliberal sólo reconoce la dimensión objetiva del trabajo, desconoce el sentido subjetivo, la vivencia personal y humana, del trabajo. Esto genera una humillación y degradación del trabajo humano.

Si se analiza desde la cultura humanista-espiritualista, el trabajo no tiene como fin exclusivo la producción sino también la subsistencia y el perfeccionamiento y desarrollo del propio trabajador. Desde esta concepción, el trabajo no sólo contribuye a la transformación de la naturaleza, a procurar el alimento o a crear nuevas tecnologías sino que también crea valores humanos, de cultura y contribuye a elevar el nivel moral y cultural de la sociedad. Desde esta óptica, el trabajo tiene una dimensión no solo económica sino también cultural. La persona que no tiene trabajo, no se realiza como ser humano y no aporta desarrollo cultural a su sociedad.

Frente a estas concepciones aparecen otras más radicales. Para Ulrich Beck ser parado y ser feliz no puede ser otra cosa que un desafió intolerable, una conciencia excesivamente lúcida, deslumbrante, un descaro futurista. Con un ingreso regular que proviniera de una fuente neutra, todas las relaciones de fuerza disimuladas y acechantes que amenazan veladamente con el despido o la degradación, saltarían por los aires. El autor define el "paro feliz" como una condición que reclama un concepto. Los parados felices son personas que están en paro y son felices y a los que les gusta estar en paro y ser feliz.

Se dice de los parados que son el rigor de las desdichas y que no desarrollan ninguna actividad que se parezca de cerca o de lejos a un trabajo. Ese es el mayor de los estereotipos sociales. El nazismo escribió a la entrada de Auschwitz "El trabajo hace libre". "El trabajo hace feliz" es hoy la ideología obligada de la sociedad del trabajo. Cuanto más atractivo pierde el trabajo, mas insisten los grandes amos de la sociedad del trabajo en que el trabajo no sólo libera sino que hace feliz.

Marx, en sus primeros escritos, ya había concebido el fin de la sociedad del trabajo: El trabajo, 
actividad totalmente ajena al hombre y a la naturaleza, por tanto a la conciencia, amenazaba con sumergir a los individuos en la nada absoluta. La vida del trabajo no era para él otra cosa que "la existencia abstracta del individuo" y afirmaba que el individuo no se sentía a sí mismo mas que fuera del trabajo.

Isabel Escudero cuestiona la necesidad y conformidad el trabajo, entendiendo por trabajo la venta de la vida, la mayoría del tiempo de nuestra vida, que ofrecemos en aras de la producción o reproducción mayormente de inutilidades, a cambio de dinero para poder comprarlas, como empujado por una especie de sacrificio o pago de una vieja deuda al parecer nunca satisfecha. Para la autora esta aceptación "natural" del trabajo para la vida, o más bien de la vida para el trabajo, supone una sumisión a la Realidad, esto es, a su falsedad constitucional.

André Gorz afirma que en Francia, entre diciembre 1997 y marzo de 1998, se ha producido una transformación y está naciendo la conciencia de que cada uno de nosotros somos precarios y parados en potencia, consiguiéndose que un $70 \%$ de la gente simpatice con el movimiento de los precarios y parados y en lugar de exigir más trabajo y situarse en una posición de dependencia respecto a la patronal, exigen una renta social suficiente durante el periodo de desempleo sin que por ello nadie pueda reprocharles pretender vivir del "trabajo de los otros".

\section{PERSPECTIVAS DE FUTURO}

Para pensar en el futuro es útil reflexionar sobre el pasado. Las condiciones de trabajo en los inicios de la industrialización fueron analizadas por Engels y Marx. Engels describe como Inglaterra tuvo un progreso colosal al situarse como centro industrial y rodearse por una serie de países agrícolas que le proporcionaban las materias primas y le compraban la mayor parte de los productos industriales que necesitaban. Cuando se liberalizó el comercio se produjo el triunfo de la clase capitalista sobre la aristocracia terrateniente. Al inicio, la clase obrera obtuvo algunas mejoras en sus condiciones generales de vida, pero con la industrialización aumentó el paro debido al uso de las máquinas y a la inmigración de los trabajadores agrícolas. Cuando Inglaterra perdió el monopolio del comercio debido a la participación de otros países, la gran masa de trabajadores sufrió inseguridad y miseria.

La historia demostró como los obreros sólo pudieron acceder a cierta independencia cuando se organizaron colectivamente. Engels señala como el movimiento obrero inglés alcanzó sus mayores logros cuando su instinto de cohesión fue tan grande que le permitió alcanzar logros electorales en 1892.

En la actualidad la crisis mundial y la precariedad del trabajo es un problema prioritario agravado por los últimos acontecimientos terroristas del 11 de septiembre de 2001. Al mismo tiempo existe un proceso de descolectivización que amenaza con acentuar la subordinación e incrementar las desigualdades sociales y son los trabajadores menos cualificados los que más sufren las consecuencias y los que menos recursos tienen para estructurar colectivos emancipatorios. Robert Castel aduce que, en un futuro próximo, no debería descartarse la posibilidad de la aparición de nuevas formas de organización.

El siglo XXI plantea muchos interrogantes en relación al trabajo del futuro. Algunos analistas piensan que el trabajo tal como es concebido en la actualidad desaparecerá. Un cambio muy importante observado desde la segunda mitad del siglo $\mathrm{XX}$, aparte de la globalización, es el crecimiento de un sistema de trabajo que no se basa en la fuerza, en la producción, sino en la información, en la mente.

\section{CONCLUSIÓN}

El empleo ha sido una de la principales preocupaciones del siglo XX como lo demuestra la creación de la Organización Internacional del Trabajo dentro de las Naciones Unidas, la puesta en marcha de políticas Keynesianas después de la gran depresión y la Carta de las Naciones Unidas. En el capítulo 55 de esta Carta se estipula que las Naciones Unidas se esforzarán por facilitar el pleno empleo. En 1966 el Pacto Internacional de derechos económicos, sociales y culturales reconoce a toda persona el derecho a trabajar, a elegir libremente un empleo, a gozar de condiciones de trabajo justas, y 
a disfrutar de la protección contra el paro. En 1995 la Cumbre Mundial para el Desarrollo Social situaba el problema del paro en el centro de los debates de la política nacional e internacional. No obstante, los valores de igualdad, libertad y solidaridad que tanto exaltan los Organismos Internacionales y los países democráticos son agredidos constantemente puesto que no es posible hablar de igualdad cuando se choca frontalmente con el incremento de las enormes desigualdades sociales, no se puede hablar de libertad cuando se niega un puesto de trabajo y se margina a grandes grupos de población impidiéndoles participar en la vida social, económica y política de su país ni se puede hablar de solidaridad cuando existe una repartición tan desquilibrada de la riqueza o cuando se priorizan los principios económicos por encima de los valores sociales.

La transición demográfica actual coincide con una profunda modificación de la organización del trabajo. Cada año entran en el mercado de trabajo 43 millones de personas más, es decir, 118.000 personas diarias. Según la Organización Internacional del Trabajo la mano de obra disponible en el mundo pasará entre 1995 y 2025 de 2.500 millones de personas a 3.700 millones y el $97 \%$ de este incremento se producirá en países que hoy están en vías de desarrollo.

¿Cómo asegurar un trabajo a la población activa actual y a los 1.200 millones de personas que habrá en el año 2.025? ¿Cómo afectará y que impacto tendrá en los diferentes grupos sociales esta situación crítica en un futuro próximo?

La precariedad laboral actual y el impacto que esta pueda tener en la salud de la población evidencian la necesidad de nuevas políticas de salud laboral y de salud pública dirigidas a los trabajadores y a sus familias, pero esto no será suficiente, ya que deberán estructurarse nuevas estrategias económicas, sociales y profundos cambios culturales respecto a la concepción del trabajo y a sus valores esenciales.

Sería falso creer que la explosión demográfica es o será la única causa de la precariedad laboral, puesto que la observación de lo que está sucediendo en los países industrializados, muestra que se mantiene o aumenta el desempleo pese a no aumentar la población e incluso existe la paradoja de producirse incremento económico sin creación de empleo.
El futuro debería dirigirse hacia la creación de un nuevo modelo de sociedad que entre todos se debería construir. Marcuse hablaba del hombre unidimensional y en las sociedades occidentales se está creando este tipo de hombre cuya necesidad más trascendente es la de poseer. Este tipo de filosofía ha asimilado la idea de que ser es igual a tener, sometiendo al ser humano a una esclavitud permanente al crearle falsas necesidades que ha de satisfacer y que no tienen otro objetivo que conseguir que los productos que continuamente aparecen en el mercado, se vendan.

Francis Fukuyama se preguntaba si las sociedades capitalistas están destinadas a ser más ricas materialmente pero más pobres moralmente. En su obra "La gran ruptura" subyace la pregunta de si el capitalismo moderno está destinado a socavar su propia base moral y a provocar su propio desmoronamiento.

La Conferencia Internacional sobre Promoción de la Salud, celebrada en Ottawa en 1986, emitió los prerrequisitos para alcanzar la salud. Afirmó que la salud es el mejor recurso para el progreso social y que los factores políticos, socioeconómicos, culturales, ambientales, comportamentales y biológicos pueden intervenir a favor o en detrimento de la salud. La precariedad laboral afecta a todas las condiciones que hacen posible la construcción de la salud por ello la Salud Pública no puede mantenerse al margen de este problema. Las funciones de defensa, capacitación y mediación de la Nueva Salud Pública deben abordar este problema porque de lo contrario la promoción de la salud y la prevención social en la comunidad no serán posibles, al menos en amplios colectivos de población.

\section{BIBLIOGRAFÍA}

- Amable M; Benach J. (2000) Crecimiento y consecuencias de la precariedad laboral. Gac. Sanit. 14(6):418-21

- André Gorz (2001) "Miserias del presente, riquezas de lo posible" en rev. Archipiélago: 57-65. Institut de Cultura. Ajuntament de Barcelona

- Boix P; Orts E; López MJ; Rodrigo F. (1997) Trabajo temporal y siniestralidad laboral en España en el periodo 1988-1995 Cuad. De Relaciones Laborales. 11:275-319 
- Castel R. (2001)¿Porqué la clase obrera ha perdido la partida? En Archipiélago; 48:37-46. Institut de Cultura. Ajuntament de Barcelona

- Castel R. (2001) ¿Porqué la clase obrera ha perdido la partida? En rev. Archipiélago: 37-46. Institut de Cultura. Ajuntament de Barcelona www.elcatalejo.com/ g_a/eeg/neeg.htm

- Comunidad de Madrid. (1999) Tasa de paro de las regiones de la UE NUTS2 wWw.comadrid.es/iestadis/ historico/ue 161001.htm

- Deniel Rosanas J; Bosch Molas M; Culi Borras N; Olmeda Brea C (1996)The influence of unemployment on mental health problems en Aten Primaria. 18(7):379-82

- Dooley D.; Prause J.; Ham-Rowbottom KA. (2000) Underemployment and depresión: longitudinal relationships en J Health Soc Beba 41(4):421-36.

- Dooley; Fielding J; Levi L. (1996) Health and unemployment: Annu Rev Public Health 17:449-65

- Dressler WW (1986)Unemployment and depressive symptoms in a southern black community en J Nerv Ment Dis 174(11):639-45

- Duran V.M. (2001) "La perspectiva de los actores sociales: El punto de vista de los trabajadores". VI Congreso Venezolano de Sociología y antropología Cultura del Trabajo y Empleo. www.utal.org/empleo2. htm

- Engels F. (1979) Situación de la clase obrera en Inglaterra (eds) Jucar Barcelona.

- François M. (1992) Le travail temporaire en Mileu Industriel. Incidences sur les Conditions de Travail et la Santé des Travailleurs en Le Travail Human 54(1):21-41

- Fukuyama F. (2000) La gran ruptura (eds). Col.leció sine qua non B. Barcelona.

- Illich I; "Needs" en Sachs W (1992) (eds) The development dicttionary: a guide to knowledge as power. Zed Books. New Jersey.

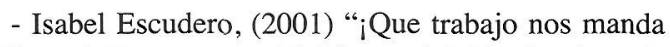
el Señor! En rev. Archipiélago: 78-80. Institut de Cultura. Ajuntament de Barcelona

- Izquierdo R (2001) Una mirada a los contratos www.lab-sindikatua.org

- Jackson T. (1999) Differences in psychosocial experiences of employed, unemployed, and student samples of young adults. J Psicol. 133(1):49-60

- Kivimaki M; Vahtera J.; Pentti J; Ferrie JE. (2000) Factors underlying the effect of organisational downsizing on health of employees: longitudinal cohort study BMJ 320:971-5

Kraut A; Mustard C; Walld R; Tate R. (2000) Unemployment and Health care utilization en Scand $\mathbf{J}$ Work Environ Health 26(2):169-177
- Marcuse H.; El hombre unidimensional (eds) (1971). Sex Barral Barcelona

- Marx K: El Capital (eds) (1978). Petronio. Barcelona

- Más Estellés J. (1997) "Una propuesta para cambiar el mundo" Rev. ANUE 10. Época IV.:28-32

- Naredo, J.M. (2001) Configuración y crisis del mito del trabajo en rev. Archipiélago 48:13-23. Institut de Cultura. Ajuntament de Barcelona

- Organización Internacional del Trabajo (1994) Empleo y población: Un dúo inseparable. Geneve

- Organización Internacional del Trabajo. Informe. (1994). Geneve

- Sahlins M; Edad de piedra, Edad de la abundancia (eds) (1977). Akal Madrid

- Siguan M. (1995) "Sobre el sentit de l’història i les tasques del nostre temps" Desequilibri nord-sud. Propostes per a reduir-lo. Ed. SECODES. Barcelona. 1: 11-21.

- Smith A. Wealth of nations (1976). citado en Naredo, J.M. Configuración y crisis del mito del trabajo en rev. Archipiélago 2001; 48:13-23. Institut de Cultura. Ajuntament de Barcelona

- Ulrich Beck (2001) "Para acabar con el imperialismo de los valores del trabajo" en rev. Archipiélago: 25-29. Institut de Cultura. Ajuntament de Barcelona

- Varcla Novo M. (1999) Unemployment and Mental health in Galicia, Spain en In Arch Occup Environ Health 72 Suppl:S14-5

- Weber M. (1998) La Ética Protestante y el Espíritu del Capitalismo (eds) ISTMO, Madrid Incorporated, Nueva York.

- Ardrey, R. (1983) La evolución del hombre. La hipótesis del cazador. Alianza $3^{\mathrm{a}}$ ed., Madrid.

- Cruz, J. (1991) Alimentación y Cultura. Antropología de la conducta alimentaria. Ed. EUNSA, Pamplona.

- Lucena, M. (1990) América 1492. Retrato de un continente hace quinientos años. Grupo Anaya S.A., Madrid.

- Farb, P y Armelagos, G (1980) Consuming Passions: The Anthropology of eating. Houghton Mifflin, Boston.

- Spector, R.E. (1985) Cultural diversity in health and illness. Appleton-Century-Crofts, Norwalk CT.

- Newberne, P.M. (1978) Diet and nutrition. Bull, New York Acad. Med. 54, 385-396, Nueva York.

- Contreras, J. (1993) Antropología de la Alimentación. Ed. Eudema, Madrid. 